\title{
Sobre propriedades de invariância de uma nova equação dispersiva
}

\author{
Igor L. Freire, \\ CMCC - Centro de Matemática, Computação e Cognição, UFABC, \\ 09210-170, Santo André, SP \\ E-mail: igor.freire@ufabc.edu.br, \\ Júlio C. S. Sampaio \\ IMECC - Depto. de Matemática Aplicada, UNICAMP, \\ 13083-859, Campinas, SP \\ E-mail: juliocesarssampaio@gmail.com.
}

Resumo: Neste trabalho estudaremos propriedades de invariância de uma família de equações dispersivas. Um dos principais objetivos é encontrar as condições para que essas equações sejam não-linearmente auto-adjuntas e calcular suas leis de conservação. Além disso, encontraremos soluções invariantes para essas equações via simetrias de Lie.

Palavras-chave: Auto-adjunticidade não-linear, leis de conservação, equações dispersivas

\section{Introdução}

No recente trabalho [4], foi estudada a seguinte família de equações dispersivas

$$
u_{t}+\frac{2 a}{u} u_{x} u_{x x}-\epsilon a u_{x x x}=0,
$$

onde $\epsilon$ é um parâmetro e $a$ uma constante. Essencialmente, podemos considerar $\epsilon$ como o coeficiente de dispersão da equação em questão.

No mesmo trabalho onde foi introduzida, algumas propriedades de tal equação foram consideradas, principalmente a construção de leis de conservação. De acordo com os resultados obtidos em [4], o caso $\epsilon=-2 / 3$ é um caso bastante especial, principalmente naquilo que tange a obtenção de leis de conservação.

Neste trabalho, estudaremos a equação (1) do ponto de vista de simetrias de Lie. De fato, mostraremos que os operadores

$$
X_{1}=\frac{\partial}{\partial t}, \quad X_{2}=\frac{\partial}{\partial x}, \quad X_{3}=u \frac{\partial}{\partial u}, \quad X_{4}=x \frac{\partial}{\partial x}+3 t \frac{\partial}{\partial t}
$$

são geradores infinitesimais de simetrias de Lie.

Uma vez encontrado o grupo de invariância, há diversas possibilidades a serem consideradas. Uma delas, que será explorada, é a obtenção de soluções invariantes, exatas, usando os geradores infinitesimais de simetrias.

Outra possibilidade, que também será abordada é a construção de leis de conservação via geradores de simetrias. Para tanto, utilizaremos os desenvolvimentos propostos por Nail Ibragimov em $[2]$.

A ideia para a construção de leis de conservação locais, introduzidas em [2], é, primeiramente, verificar se a equação considerada é não-linearmente auto-adjunta, veja [3]. Dessa forma, se 
positiva a questão, é possível, a partir dos geradores de simetrias, encontrar leis de conservação locais.

Na próxima seção mostraremos que tal equação é não-linearmente auto-adjunta, o que nos possibilitará encontrar leis de conservação locais utilizando [2].

\section{Leis de conservação}

"Na natureza nada se perde, nada se cria, tudo se transforma". Com essas palavras, Antoine Lavoisier enunciou a lei de conservação de massa, que diz que numa reação química, a quantidade de reagentes deve ser igual a quantidade de produtos.

Conhecemos outras leis de conservação frequentemente utilizadas, como a lei de conservação de energia, do momento linear e do momento angular.

Mas o que é uma lei de conservação? Basicamente uma lei de conservação é um balanceamento de alguma substância concreta ou abstratatamente. Em termos matemáticos, uma lei de conservação para uma EDP é um vetor cuja divergência se anula nas soluções dessa EDP.

Geralmente não é uma tarefa simples encontrar uma lei de conservação para uma EDP. Um resultado clássico conhecido para encontrar leis de conservação é o Teorema de Noether, que fornece um algoritimo para calcular leis de conservação via simetrias de Lie-Bäcklund. Tal resultado é entretanto restritivo, no sentido de que só pode ser aplicada a equações diferenciais advindas de uma integral de ação, e consequentemente essas equações diferenciais sempre são de ordem par.

Em 2009 Ibragimov propôs a seguinte definição em seu trabalho, veja [2].

Seja

$$
F\left(x, u, u_{(1)}, \ldots, u_{(k)}\right)=0
$$

uma equação diferencial parcial. A equação

$$
F^{*}\left(x, u, v, u_{(1)}, v_{(1)}, \ldots, u_{(k)}, v_{(k)}\right)=\frac{\delta}{\delta u} \mathcal{L}=0
$$

é chamada equação adjunta à $F\left(x, u, u_{(1)}, \ldots, u_{(k)}\right)=0$, onde

$$
\frac{\delta}{\delta u}=\frac{\partial}{\partial u}-D_{i} \frac{\partial}{\partial u_{i}}+D_{i} D_{j} \frac{\partial}{\partial u_{i j}}+\ldots(-1)^{k} D_{i_{1}} \ldots D_{i_{k}} \frac{\partial}{\partial u_{i_{1} \ldots i_{k}}},
$$

é o operador de Euler-Lagrange e $\mathcal{L}=v F\left(x, u, u_{(1)}, \ldots, u_{(k)}\right)$ é chamada de lagrangeana formal, onde $v=v(x)$ é uma nova variável dependente.

No mesmo trabalho, Ibragimov provou que o vetor

$$
\begin{aligned}
C^{i}= & \xi^{i} \mathcal{L}+W\left[\frac{\partial \mathcal{L}}{\partial u_{i}}-D_{j}\left(\frac{\partial \mathcal{L}}{\partial u_{i j}}\right)+D_{j} D_{k} \frac{\partial \mathcal{L}}{\partial u_{i j k}}-\cdots\right] \\
& +D_{j}(W)\left[\frac{\partial \mathcal{L}}{\partial u_{i j}}-D_{k}\left(\frac{\partial \mathcal{L}}{\partial u_{i j k}}\right)+\cdots\right] \\
& +D_{j} D_{k}(W)\left[\frac{\partial \mathcal{L}}{\partial u_{i j k}}-\cdots\right]+\cdots
\end{aligned}
$$

e $W=\eta-\xi^{i} u_{i}$, é um vetor conservado para o sistema de equações formado pela EDP e sua respectiva equação adjunta, onde $x=\left(x_{1}, x_{2}, \ldots, x_{n}\right) \in \Omega \subset \mathbb{R}^{n}, u=u(x)$ e $u_{(k)}$ o conjunto de todas as derivadas parciais de $k$-ésima ordem de $u$. Também está sendo usada a conveção da soma de Einstein, onde índices repetidos devem ser somados.

Este resultado de Ibragimov é em certo sentido mais geral, uma vez que pode ser aplicada a uma EDP qualquer, lembrando entretanto que os vetores conservados são agora para o sistema de equações e não para a EDP propriamente dita.

Em 2011 Ibragimov [3] desenvolveu a seguinte definição: 
Definição 1. Uma EDP é dita ser não-linearmente auto-adjunta, se a seguinte condição é satisfeita:

$$
\left.F^{*}\left(x, u, v, u_{(1)}, v_{(1)}, \ldots, u_{(k)}, v_{(k)}\right)\right|_{v=\phi(x, u)}=\lambda F\left(x, u, u_{(1)}, \ldots, u_{(k)}\right),
$$

onde $\lambda=\lambda(x, u, \ldots)$ e $\phi(x, u) \neq 0$. A função $\phi$ que satisfaz (4) é chamada substituição.

Se uma EDP

$$
F\left(x, u, u_{(1)}, \ldots, u_{(k)}\right)=0
$$

é não-linearmente auto-adjunta, (3) é um vetor conservado para $F\left(x, u, u_{(1)}, \ldots, u_{(k)}\right)=0$, quando substituímos $v=\phi(x, u)$ em (3).

Exemplo 1. A equação

$$
u_{t}+u u_{x x x}=0 .
$$

é não-lineramente auto-adjunta. A expressão para $\phi$ é dada por

$$
\phi(x, t, u)=c_{1}\left(\frac{x^{3}}{u}-6 t\right)+c_{2} \frac{x^{2}}{u}+c_{3} \frac{x}{u}+\frac{c_{4}}{u}+c_{5},
$$

veja [1].

Considerando o gerador de simetrias de Lie

$$
X=t \frac{\partial}{\partial t}-u \frac{\partial}{\partial u}
$$

as componentes dadas por (3) nos fornece

$$
\begin{aligned}
C^{0}= & v\left(t u u_{x x x}-u\right) \\
C^{1}= & v\left(-2 u u_{x x}+u_{x}^{2}+t u_{x} u_{x t}-t u u_{x x t}-t u_{t} u_{x x}\right) \\
& +v_{x}\left(t u u_{x t}-2 t u_{x} u_{t}-u u_{x}\right)-v_{x x}\left(u^{2}+t u u_{t}\right) .
\end{aligned}
$$

Substituindo $v=1$ em (7) e após alguns calculos, temos

$$
\begin{aligned}
C^{0} & =-u-D_{x}\left(\frac{t u_{x}^{2}}{2}-t u u_{x x}\right), \\
C^{1} & =-u u_{x x}+\frac{u_{x}^{2}}{2}+D_{t}\left(\frac{t u_{x}^{2}}{2}-t u u_{x x}\right) .
\end{aligned}
$$

Então, transferindo os termos $D_{t}(\cdots)$ de $C^{0}$ e $C^{1}$, obtemos as seguintes expressões

$$
C^{0}=u, C^{1}=u u_{x x}-\frac{u_{x}^{2}}{2} .
$$

Vamos verificar que (1) é não linearmente auto-adjunta. Usando o Teorema 2 de [1], obtemos o seguinte sistema de equações

$$
\begin{gathered}
\phi_{t}-\epsilon a \phi_{x x x}=0, \\
\frac{2}{u} \phi_{x}+3 \epsilon \phi_{x u}=0, \\
\epsilon a \phi_{x u u}=0,
\end{gathered}
$$




$$
2\left(\frac{\phi}{u}\right)_{u}+\epsilon \phi_{u u}=0
$$

Da equação (10) (supondo $a \neq 0$ e $\epsilon \neq 0$ ) temos

$$
\begin{gathered}
\phi_{x u u}=0 \Rightarrow\left(\phi_{x}\right)_{u u}=0 \Rightarrow \phi_{x}=A(x, t) u+B(x, t) \\
\phi(x, t, u)=\psi(x, t) u+\varphi(x, t)+\rho(t, u),
\end{gathered}
$$

onde $\psi(x, t)=\int A(x, t) d x$ e $\varphi(x, t)=\int B(x, t) d x$.

Substituindo (12) em (10), temos

$$
\begin{gathered}
\left(1+\frac{3}{2} \epsilon\right) \psi_{x}=0, \\
\varphi_{x}=0,
\end{gathered}
$$

Da equação (14) concluímos que $\varphi=\varphi(t)$. A partir da equação (13), podemos considerar dois casos. O primeiro é quando $\epsilon \neq-\frac{2}{3}$, neste caso devemos ter $\psi_{x}=0$ e logo $\psi=\psi(t)$. Portanto

$$
\phi(x, t, u)=\phi(t, u)=\psi(t) u+\varphi(t)+\rho(t, u) .
$$

Substituindo (15) em (9), obtemos $\psi^{\prime} u+\varphi^{\prime}+\rho_{t}=0$ o que implica em

$$
\frac{\partial}{\partial t}(\psi(t) u+\varphi(t)+\rho(t, u))=0 .
$$

Assim $\psi(t) u+\varphi(t)+\rho(t, u)=k(u)$, ou seja, $\phi=\phi(u)$. Então a partir da equação (11) obtemos a seguinte EDO

$$
u^{2} k^{\prime \prime}+\frac{2}{\epsilon} u k^{\prime}-\frac{2}{\epsilon} k=0
$$

cuja solução é

$$
k(u)=c_{1} u+c_{2} u^{-\frac{2}{\epsilon}},
$$

se $\epsilon \neq-2$. Se $\epsilon=-2$, temos a seguinte solução para (16)

$$
k(u)=c_{1} u+c_{2} \ln (u) u .
$$

Portanto concluímos que $\phi(u)=c_{1} u+c_{2} u^{-\frac{2}{\epsilon}}$ quando $\epsilon \neq-2$ e $\phi(u)=c_{1} u+c_{2} \ln (u) u$ quando $\epsilon=-2$, onde $c_{1}$ e $c_{2}$ são constantes reais.

Se $\epsilon=-2 / 3$, a partir da equação (11), obtemos

$$
u^{2} \rho_{u u}-3 u \rho_{u}+3 \rho=-3 \varphi .
$$

Para cada $t$ fixo e arbitrário, a equação (19) é uma EDO em $u$, onde a solução geral é dada por

$$
\rho(t, u)=k_{1}(t) u+k_{2}(t) u^{3}-\varphi(t) .
$$

Sunstituindo (20) na expressão de $\phi$, obtemos

$$
\phi(x, t, u)=\left(\psi(x, t)+k_{1}(t)\right) u+k_{2}(t) u^{3},
$$


A partir da equação (8), concluímos que

$$
\left(\psi_{t}+\frac{2}{3} a \psi_{x x x}+k_{1}^{\prime}\right) u+k_{2}^{\prime} u^{3}=0
$$

logo $k_{2}^{\prime}=0$ o que implica que $k_{2}(t)=c=$ constante e $\psi$ e $k_{1}$ cumprem a seguinte EDP

$$
\psi_{t}+\frac{2}{3} a \psi_{x x x}+k_{1}^{\prime}=0
$$

Neste trabalho, além da classificação em relação à auto-adjunticidade não-linear, também encontramos, utilizando os geradores de simetrias de Lie (2) as correspondentes leis de conservação para a equação (1).

\section{Agradecimentos}

Os autores agradecem à FAPESP pelo suporte financeiro, através do processo $n^{\circ}$ 2011/23538-0. I. L. Freire agradece à FAPESP, processo no 2011/19089-6 e ao CNPQ, processo no $308941 / 2013-$ 6.

\section{Referências}

[1] I. L. Freire and J. C. Santos Sampaio, On the nonlinear self-adjointness and local conservation laws for a class of evolution equations unifying many models, Commun. Nonlinear. Sci. Numer. Simul., 19, (2014) 350-360.

[2] N. H. Ibragimov, A new conservation theorem, J. Math. Anal. Appl., 333, (2007) 311-328.

[3] N. H. Ibragimov, Nonlinear self-adjointness and conservation laws, J. Phys. A Math. Theor., 44 (2011) 8pp.

[4] A. Sen, D. P. Ahalpara, A. Thyagaraja and G. S. Krishnaswami, A KdV-like advectiondispersion equation with some remarkable properties, Commun. Nonlinear. Sci. Numer. Simul., 17, (2012) 4115-4124. 